\section{Dramatically improved survival in multiple myeloma patients in the recent decade: results from a Swedish population-based study}

Multiple myeloma (MM) is generally considered an incurable disease. In recent decades, new treatment alternatives have changed both the clinical course of the disease and improved survival. ${ }^{1}$ Results from previous population-based studies in Sweden for patients diagnosed until 2003 have shown an increased survival rate in patients up to 70 years old at diagnosis, ${ }^{2}$ and studies from the USA have shown improved long-term survival in patients between 65 to 80 years old at diagnosis, and reduced early mortality in all age groups in recent years. ${ }^{3}$ Subsequently, clinical trials have shown that using novel agents as first-line treatment can further improve survival in $\mathrm{MM} .{ }^{4}$ However, there remains the unanswered question of how the use of immunomodulatory drugs and proteasome inhibitors as first-line treatment of $\mathrm{MM}$ affects survival in a large population-based patient cohort. Real-world data, such as population-based stud- ies, are very important tools to evaluate this, since the included participants in clinical trials, for the most part, are generally not representative of the whole MM patient group, which compromises their generalizability. ${ }^{5}$

We performed a large population-based study using the Swedish Cancer Register and included 21,502 MM patients diagnosed over a 40-year period in order to define changes in survival in all patients with $\mathrm{MM}$ from 1973 to 2013.

Patients diagnosed with $\mathrm{MM}$ in the period from January $1^{\text {st }} 1973$ to December $31^{\text {st }} 2013$ were identified from the Swedish Cancer Register, a centralized, nationwide register that contains information, going back to 1958 , on patients diagnosed with malignant disorders in Sweden. Information on sex, date of birth, date of diagnosis, and date of death were collected. Information on clinical stage and laboratory results were not available for these patients.

Relative survival ratios (RSRs) were used to provide a measure for the excess mortality of MM patients in relation to a comparable group from the general population, irrespective of whether the excess mortality is directly or

Table 1. Relative survival ratios for patients diagnosed with multiple myeloma in Sweden, stratified by age group and calendar period at diagnosis.

\begin{tabular}{|c|c|c|c|c|}
\hline Age at diagnosis & $\begin{array}{c}\text { RSR (95\% CI) } \\
1973-1982\end{array}$ & $\begin{array}{c}\text { Calendar Period } \\
\text { RSR (95\% Cl) } \\
1983-1992\end{array}$ & $\begin{array}{c}\text { RSR (95\% CI) } \\
1993-2002\end{array}$ & $\begin{array}{c}\text { RSR (95\% Cl) } \\
2003-2013\end{array}$ \\
\hline \multicolumn{5}{|l|}{ Three-month RSRs } \\
\hline $20-40$ & 0.94 (0.82 to 0.98$)$ & 0.98 (0.87 to 1.00$)$ & 0.96 (0.83 to 0.99$)$ & 0.98 (0.88 to 1.00$)$ \\
\hline $41-50$ & 0.95 (0.90 to 0.97$)$ & 0.96 (0.93 to 0.98$)$ & 0.98 (0.96 to 0.99$)$ & 0.98 (0.95 to 0.99$)$ \\
\hline $51-60$ & 0.90 (0.88 to 0.92$)$ & 0.94 (0.91 to 0.95$)$ & 0.96 (0.94 to 0.97$)$ & 0.98 (0.97 to 0.99$)$ \\
\hline $61-70$ & 0.87 (0.85 to 0.89$)$ & $0.92(0.91$ to 0.94$)$ & 0.93 (0.92 to 0.94$)$ & 0.96 (0.95 to 0.97) \\
\hline $71-80$ & 0.81 (0.79 to 0.83$)$ & 0.87 (0.86 to 0.89$)$ & 0.88 (0.86 to 0.89$)$ & 0.92 (0.91 to 0.94$)$ \\
\hline$>80$ & 0.68 (0.64 to 0.72$)$ & 0.76 (0.73 to 0.79$)$ & 0.81 (0.78 to 0.83$)$ & 0.84 (0.82 to 0.86$)$ \\
\hline \multicolumn{5}{|l|}{ One-year RSRs } \\
\hline $20-40$ & 0.92 (0.79 to 0.97$)$ & 0.92 (0.81 to 0.97$)$ & 0.91 (0.78 to 0.97$)$ & 0.92 (0.81 to 0.97) \\
\hline $41-50$ & 0.82 (0.75 to 0.87$)$ & 0.86 (0.81 to 0.90$)$ & $0.94(0.90$ to 0.96$)$ & 0.93 (0.89 to 0.96$)$ \\
\hline $51-60$ & 0.75 (0.72 to 0.78$)$ & 0.84 (0.81 to 0.87$)$ & 0.91 (0.88 to 0.93$)$ & $0.92(0.90$ to 0.94$)$ \\
\hline $61-70$ & $0.74(0.72$ to 0.76$)$ & 0.80 (0.77 to 0.82$)$ & 0.84 (0.82 to 0.86$)$ & 0.88 (0.86 to 0.90$)$ \\
\hline $71-80$ & 0.66 (0.63 to 0.68$)$ & 0.73 (0.71 to 0.75$)$ & 0.73 (0.70 to 0.75$)$ & 0.80 (0.79 to 0.82$)$ \\
\hline$>80$ & 0.49 (0.45 to 0.54$)$ & 0.55 (0.51 to 0.59$)$ & 0.62 (0.58 to 0.65$)$ & 0.66 (0.63 to 0.69$)$ \\
\hline \multicolumn{5}{|l|}{ Five-year RSRs } \\
\hline $20-40$ & 0.48 (0.34 to 0.62$)$ & 0.57 (0.43 to 0.70$)$ & 0.67 (0.51 to 0.78$)$ & 0.59 (0.43 to 0.72$)$ \\
\hline $41-50$ & 0.47 (0.39 to 0.55$)$ & $0.50(0.43$ to 0.56$)$ & 0.61 (0.55 to 0.67$)$ & 0.70 (0.62 to 0.76$)$ \\
\hline $51-60$ & $0.36(0.33$ to 0.40$)$ & $0.44(0.40$ to 0.48$)$ & 0.56 (0.52 to 0.59$)$ & $0.62(0.58$ to 0.66$)$ \\
\hline $61-70$ & 0.31 (0.29 to 0.34$)$ & 0.34 (0.32 to 0.37$)$ & $0.36(0.33$ to 0.39$)$ & 0.48 (0.45 to 0.51$)$ \\
\hline $71-80$ & 0.24 (0.21 to 0.26$)$ & $0.28(0.26$ to 0.31$)$ & $0.24(0.22$ to 0.26$)$ & 0.34 (0.31 to 0.37$)$ \\
\hline$>80$ & 0.14 (0.10 to 0.18$)$ & 0.14 (0.11 to 0.17$)$ & 0.16 (0.13 to 0.19$)$ & 0.15 (0.12 to 0.19$)$ \\
\hline \multicolumn{5}{|l|}{ Ten-year RSRs } \\
\hline $20-40$ & 0.38 (0.25 to 0.52$)$ & 0.30 (0.18 to 0.43$)$ & 0.48 (0.33 to 0.62$)$ & 0.45 (0.28 to 0.61$)$ \\
\hline $41-50$ & 0.21 (0.15 to 0.28$)$ & $0.31(0.25$ to 0.38$)$ & 0.39 (0.32 to 0.45$)$ & 0.41 (0.29 to 0.54$)$ \\
\hline $51-60$ & 0.16 (0.13 to 0.19$)$ & 0.18 (0.15 to 0.22$)$ & 0.28 (0.24 to 0.31$)$ & 0.36 (0.30 to 0.43$)$ \\
\hline $61-70$ & 0.11 (0.09 to 0.13$)$ & $0.14(0.12$ to 0.16$)$ & 0.14 (0.12 to 0.16$)$ & $0.22(0.18$ to 0.27$)$ \\
\hline $71-80$ & 0.06 (0.05 to 0.08$)$ & 0.08 (0.07 to 0.10$)$ & 0.08 (0.06 to 0.10$)$ & 0.13 (0.09 to 0.17$)$ \\
\hline$>80$ & 0.05 (0.02 to 0.12$)$ & 0.05 (0.03 to 0.10$)$ & 0.02 (0.01 to 0.05$)$ & 0.08 (0.04 to 0.17$)$ \\
\hline
\end{tabular}

RSR: relative survival ratio; CI: confidence interval. 
indirectly associated with the disease. ${ }^{6}$ RSR is defined as the observed survival in the patient group divided by the expected survival of the general population. The Ederer II method was used to estimate the expected survival in the Swedish population life-tables, stratified by age, sex, and calendar time. RSRs with $95 \%$ confidence intervals (CIs) were estimated for one, five and ten years for four calendar periods; 1973-1982, 1983-1992, 1993-2002 and 2003-2013, and furthermore for six age categories (20-40, 41-50, 51-60, 61-70, 71-80 and >80). Short-term survival, as defined by RSR for the first three months after MM diagnosis was also defined for all calendar periods.

Additionally, we modeled relative survival using flexible parametric relative survival models ${ }^{7}$ with time since diagnosis modeled using natural splines with five degrees of freedom. Age at diagnosis and calendar period were modeled as categorical variables in some models to produce tables of hazard ratios, but also modelled as natural splines, each with four degrees of freedom, to illustrate temporal trends in relative survival as smooth flexible functions.

In the period from January $1^{\text {st }} 1973$ to December $31^{\text {st }}$ 2013 , a total of 21,502 patients with $\mathrm{MM}$ were reported to the Swedish Cancer Register. Males constituted 11,655 (54\%) and females 9847 (46\%). Overall, three-month, one-year, five-year, and ten-year RSR improved in MM patients diagnosed from 1973 to 2013 (Figure 1). The five-year RSR also improved in all four calendar periods, although the improvement was not significant between the two first periods (the RSRs were 0.28 (95\% CI: 0.27 0.30), 0.31 (95\% CI: 0.30-0.33), 0.33 (95\% CI: 0.31-0.34), and 0.41 (95\% CI: $0.40-0.43$ ), respectively). Similarly, analysis of the Surveillance, Epidemiology, and End Results 1975-2010 USA database, showed an improvement of five-year survival in patients diagnosed between 1999-2005. ${ }^{8}$ A European multi-center study likewise found that relative survival increased from $30 \%$ to $40 \%$ between 1997 and 1999 and 2006 and 2008. ${ }^{\circ}$ The most likely explanation for increased survival in the third calendar period in our study is widespread treatment with high-dose melphalan/autologous transplantation (HDM/ASCT), introduced in the 1980's, which was used in many of the younger patients from 1994 on, at the least. ${ }^{2}$ In Sweden, around $80 \%$ of patients under 65 years at diagnosis received HDM/ASCT in 2008-2012. ${ }^{10}$ In the last calendar period, the effect of thalidomide, lenalido-

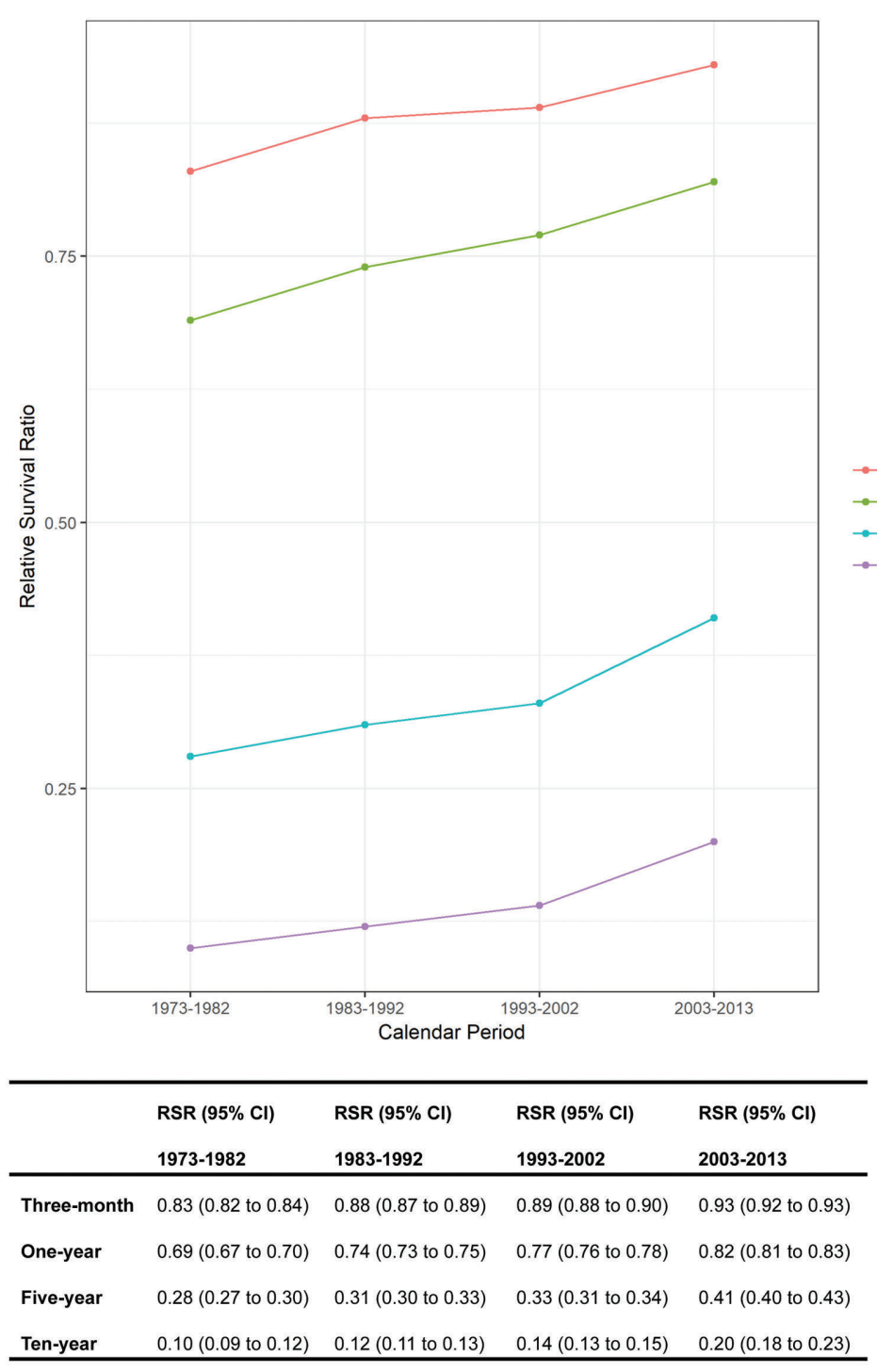

Figure 1. Relative survival ratios (RSRs) for patients diagnosed with multiple myeloma in Sweden, stratified by calendar period. RSR: relative survival ratio; $\mathrm{Cl}$ : confidence interval. 


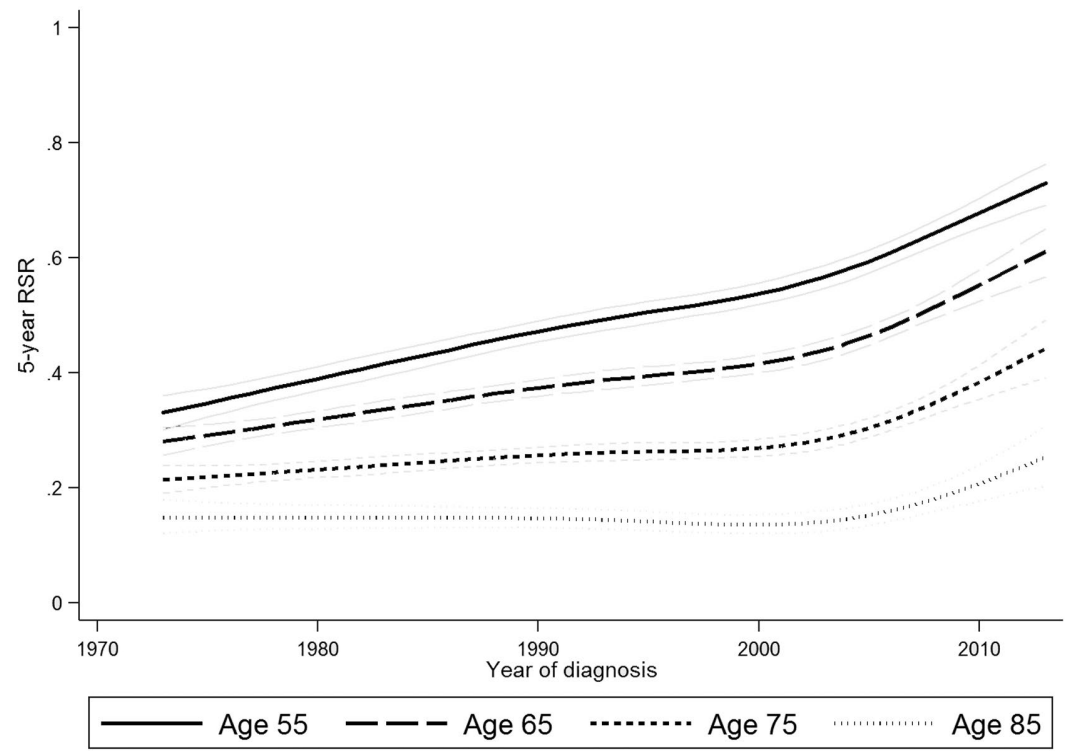

Figure 2. Model-based estimates of fiveyear relative survival ratios for four selected values of age. RSR: relative survival ratio. mide and bortezomib is the most likely explanation of increased survival, since thalidomide was introduced in MM treatment in 1999 and bortezomib and lenalidomide in the early 2000 s. $^{2,10}$ In the subsequent years, these new agents were used more frequently as first-line treatment of MM. ${ }^{11}$ From 2008 to 2012 the proportion of patients receiving thalidomide, bortezomib or lenalidomide as a part of first-line treatment increased from $29 \%-68 \%$ for all patients in Sweden; this was even higher for the patients under 65 years old in $2012(87 \%){ }^{10}$

The ten-year RSR did not improve significantly between the first three calendar periods, but increased significantly between the two last calendar periods, the RSRs being 0.10 (95\% CI: 0.09-0.12), 0.12 (95\% CI: $0.11-$ $0.13), 0.14$ (95\% CI: 0.13-0.15) and 0.20 (95\% CI: 0.180.23), respectively, (Figure1). This is consistent with results from clinical trials which have shown increasing long-term survival, which has led authors to suggest that MM could soon be classified as either a curable or chronic disease. ${ }^{12}$

Three-month RSR increased in MM patients diagnosed during the study period, although the improvement was not significantly increased between the second and third calendar periods, and in the four calendar periods resulted as 0.83 (95\% CI: 0.82-0.84), 0.88 (95\% CI: 0.87-0.89), 0.89 (95\% CI: 0.88-0.90), and 0.93 (95\% CI: $0.92-0.93$ ), respectively. The one-year RSR increased significantly between all calendar periods, and was 0.69 (95\% CI: 0.67-0.70), 0.74 (95\% CI: 0.73-0.75), 0.77 (95\% CI: 0.760.78 ), and 0.82 (95\% CI: $0.81-0.83$ ) in the four calendar periods, respectively. The three-month RSR increased in all age groups from the first to the last calendar period, although the increase was not significant in the two youngest age groups (20-40 and 41-50 years at diagnosis; Table 1). One-year RSR was significantly increased between the first and last calendar periods for all age groups, except that of the youngest group where the RSR did not change significantly. Similarly, in a recent study from the USA, early mortality was higher between 2005 and 2010, compared to the rate between 2000 and 2005, and early mortality was significantly lower in patients receiving a novel agent as part of the initial treatment. ${ }^{13}$ Other possible contributing factors to improved short- term survival include: early and aggressive treatment of renal failure, hypercalcemia, infections, and venous thromboembolism prophylaxis. ${ }^{14}$ Another consideration is that patients may have been diagnosed at an earlier stage of disease, although no data, to our knowledge, has demonstrated this.

The five-year and ten-year RSR increased significantly between the first and last calendar periods for all agegroups except for the youngest (20-40 years at diagnosis) and the oldest group ( $>80$ years old at diagnosis) where the RSRs were similar (Table 1). In elderly patients, we found that the five-year survival rate in the largest age group, 71-80 years old at the time of diagnosis, increased significantly between the last two calendar periods. Furthermore, the ten-year relative survival for the same age group was significantly increased in the last calendar period, as compared to the first period. This is consistent with a population-based study from the USA, where survival in MM patients diagnosed between 1973 and 2009 was found to be improved, over time, in all age groups up to 80 years of age. ${ }^{3}$ It is, however, a point of concern that there is no evidence of improvement in long-term relative survival in patients over 80 years old at diagnosis during the 40 years of the study. Although this age group represents $18 \%$ of all MM patients in our data, and is expected to increase in number over time, limited data exist on optimal treatment for these patients. Even in studies focusing on "elderly" patients, those patients over 80 years old at the time of diagnosis are often not included. ${ }^{5}$ Therefore, in this group of patients, which are clearly not benefitting from novel treatment options, more studies are needed to detangle the underlying explanation, along with clinical trials focusing on this large group of often frail patients.

Our study has several strengths, including the large population-based design with high-quality data, including all MM patients reported to the Swedish Cancer Register during a period of 40 years. ${ }^{15}$ Furthermore, the collection of data was carried out prospectively, independent of our study. Limitations of the study herein include lack of clinical data for individual patients, including clinical stage of disease or the treatment each patient received. This could improve to lead time bias if $\mathrm{MM}$ is 
detected at an earlier stage in the later calendar-periods.

In conclusion, our large population-based study showed that the survival of MM patients has been improving dramatically, in particular during the last decade. We believe this progress is due to the revolutionary changes in the therapeutic arsenal and supportive care in MM. Further efforts are nevertheless needed for the oldest patients, although the impact of newer treatments is spreading to older age groups. Through ongoing research and the introduction of agents with novel mechanisms of action, survival will hopefully continue to improve in the coming years, as we make advances towards changing $\mathrm{MM}$ to a chronic or a curable condition.

Sigrun Thorsteinsdottir, ${ }^{1,2}$ Paul W. Dickman, ${ }^{3}$ Ola Landgren, ${ }^{4}$ Cecilie Blimark, ${ }^{5}$ Malin Hultcrantz, ${ }^{4,6}$ Ingemar Turesson, ${ }^{7}$ Magnus Björkholm ${ }^{6}$ and Sigurdur Y. Kristinsson ${ }^{1,2,6}$

${ }^{1}$ Department of Internal Medicine, Landspitali - The National University Hospital of Iceland, Reykjavik, Iceland; ${ }^{2}$ Faculty of Medicine, University of Iceland, Reykjavik, Iceland; ${ }^{3}$ Department of Medical Epidemiology and Biostatistics, Karolinska Institutet, Stockholm, Sweden; ${ }^{4}$ Myeloma Service, Division of Hematologic Oncology, Memorial Sloan-Kettering Cancer Center, NY, USA; 'Department of Internal Medicine, Hematology Section, Sahlgrenska University Hospital, Gothenburg, Sweden; 'Department of Medicine, Division of Hematology, Karolinska University Hospital and Karolinska Institutet, Stockholm, Sweden and 'Department of Hematology and Coagulation Disorders, Skane University Hospital, Malmo, Sweden

Funding: this research was supported by grants from the University of Iceland Research Fund, Icelandic Centre for Research (RANNIS), Landspitali University Hospital Research Fund, Karolinska Institutet Foundations, and Marie Curie CIG

Correspondence:sigyngvi@hi.is doi:10.3324/haematol.2017.183475

Information on authorship, contributions, and financial \& other disclosures was provided by the authors and is available with the online version of this article at www. haematologica.org

\section{References}

1. Palumbo A, Anderson K. Multiple myeloma. N Engl J Med. 2011;364(11):1046-1060.
2. Kristinsson SY, Landgren O, Dickman PW, et al. Patterns of survival in multiple myeloma: a population-based study of patients diagnosed in Sweden from 1973 to 2003. J Clin Oncol. 2007;25(15):19931999.

3. Kristinsson SY, Anderson WF, Landgren O. Improved long-term survival in multiple myeloma up to the age of 80 years. Leukemia. 2014;28(6):1346-1348.

4. Palumbo A, Rajkumar SV, San Miguel JF, et al. International Myeloma Working Group consensus statement for the management, treatment, and supportive care of patients with myeloma not eligible for standard autologous stem-cell transplantation. J Clin Oncol. 2014;32(6):587-600.

5. Costa LJ, Hari PN, Kumar SK. Differences between unselected patients and participants in multiple myeloma clinical trials in US: a threat to external validity. Leuk Lymphoma. 2016;57(12):2827-2832.

6. Dickman PW, Adami HO. Interpreting trends in cancer patient survival. J Intern Med. 2006;260(2):103-117.

7. Lambert PC, Royston P. Further development of flexible parametric models for survival analysis. Stata J. 2009;9:265-290.

8. Bergsagel PL. Where we were, where we are, where we are going: progress in multiple myeloma. Am Soc Clin Oncol Educ Book. 2014;199-203.

9. Sant M, Minicozzi P, Mounier M, et al. Survival for haematological malignancies in Europe between 1997 and 2008 by region and age: results of EUROCARE-5, a population-based study. Lancet Oncol. 2014;15(9):931-942.

10. Blimark $\mathrm{CH}$, Turesson I, Genell A, et al. Outcome and survival of myeloma patients diagnosed 2008-2015. Real world data on 4904 patients from the Swedish Myeloma Registry (SMR). Haematologica. 2017 Dec 7. [Epub ahead of print]

11. Blimark C, Kristinsson SY, Genell A. Myelom, Nationell rapport för anmälningar 2008-2013 och ett-års uppföljning av 2008-2012 från Nationellt kvalitetsregister för myelom. 2015.

12. San-Miguel JF, Mateos MV. Can multiple myeloma become a curable disease? Haematologica. 2011;96(9):1246-1248.

13. Kumar SK, Dispenzieri A, Lacy MO, et al. Continued improvement in survival in multiple myeloma: changes in early mortality and outcomes in older patients. Leukemia. 2014;28(5):1122-1128.

14. Gonsalves WI, Godby K, Kumar SK, et al. Limiting early mortality: do's and dont's in the management of patients with newly diagnosed multiple myeloma. Am J Hematol. 2016;91(1):101-108.

15. Turesson I, Linet MS, Bjorkholm M, et al. Ascertainment and diagnostic accuracy for hematopoietic lymphoproliferative malignancies in Sweden 1964-2003. Int J Cancer. 2007;121(10):2260-2266. 